\title{
Use of self expanding stents in stenotic aortopulmonary shunts in adults with complex cyanotic heart disease
}

\author{
R Bader, J Somerville, A Redington
}

\begin{abstract}
Objective-To describe the use of self expanding stents in treating long segment stenosis of aortopulmonary shunts (APS) in adults.

Design-Clinical records, catheterisation data, cineangiograms, and operation notes of four consecutive patients undergoing stent implantation since December 1994 were studied retrospectively.

Setting-A tertiary referral centre for cardiac disease.

Subjects-Four patients underwent cardiac catheterisation because of clinical deterioration. Their age ranged between 23 and 32 years. The underlying diagnosis was complex cyanotic heart disease in all. Three had a stenotic interposition graft, and one had a classic Blalock shunt.

Results-There was one technical failure owing to migration of the stent distal to an ostial stenosis. The ability index, resting oxygen saturation, and exercise tolerance improved in the remainder. Their medium term results have been excellent.

Conclusions-This technique may further palliate adult patients with complex congenital heart disease, though the long term patency of stents is unknown.

(Heart 1999;82:27-29)
\end{abstract}

Keywords: congenital heart disease; stents; aortopulmonary shunt

Paediatrics, Royal

Brompton Hospital and National Heart and Lung Institute

A Redington

Correspondence to: Dr Redington.

Accepted for publication 1 February 1999 the need for demanding surgical intervention. The use of balloon expandable stents in occluded or stenosed Blalock-Taussig shunts has been reported in a small number of infants. ${ }^{34}$ In this report we describe the use of self expanding stents, which may have inherent advantages, particularly when treating long segment stenosis of multiply stenotic vessels in older patients.

\section{Methods}

PATIENTS

Four patients undergoing cardiac catheterisation to investigate increasing cyanosis were included in the study. Their underlying condition, previous surgery, and anthropometric data are given in table 1 .

All had previously undergone at least one systemic arterial to pulmonary artery shunt procedure: one classical Blalock-Taussig shunt, two modified (Impra graft) Blalock-Taussig shunts, and one central ascending aortic to left pulmonary artery shunt. Patients were considered for stent implantation if they had stenotic shunt lesions which could not be relieved adequately by standard balloon dilatation.

\section{TECHNICAL DETAILS}

The technical details were the same in each case and similar to those described previously. ${ }^{5}$ Cardiac catheterisation was performed under general anaesthesia in all. Following the diagnostic part of the procedure, a retrograde arterial catheter was passed through the stenotic systemic arterial to pulmonary arterial shunt. A 0.035 inch exchange length guide wire was placed in the distal pulmonary arterial tree. The desired length of the self expanding stent was assessed by withdrawing the indwelling catheter across the area to be stented, taking the distance removed at the groin as the desired length of the deployed stent. The desired diameter was assessed either from the original size of the implanted prosthetic material, or the desired size of the communication (in the patient with the classic Blalock shunt). The self expanding stent delivery system (WallstentSchneider) was passed through the indwelling femoral arterial sheath retrogradely into the shunt. It was then deployed in the usual way ${ }^{5}$ taking care that both the distal and proximal ends were in the ideal position. In general, the distal end of the stent was opened distal to its ultimately desired position, and when $50 \%$ or so of the device had been deployed it was pulled back into optimal position. The delivery system was then withdrawn and repeat

AOP, age at operation in years; BTS, Blalock-Taussig shunt; CPA, complex pulmonary atresia; DILV, double inlet left ventricle; PS, pulmonary stenosis; RV, right ventricle; TGA, transposition of great vessels; TV, tricuspid valve; VSD, ventricular septal defect; VA, ventriculoarterial. 
Table 2 Procedural results with outcome data

\begin{tabular}{|c|c|c|c|c|c|c|c|c|c|c|}
\hline \multirow[b]{2}{*}{ Case } & \multicolumn{4}{|l|}{ Before stent } & \multicolumn{4}{|l|}{ After stent } & \multirow[b]{2}{*}{$\begin{array}{l}\text { Stent } \\
\text { size }\end{array}$} & \multirow[b]{2}{*}{$\begin{array}{l}F U \\
\text { (years) }\end{array}$} \\
\hline & $\begin{array}{l}\text { Resting } \mathrm{O}_{2} \\
\text { sat (\%) }\end{array}$ & $\begin{array}{l}E T(\mathrm{~min}) / \mathrm{O}_{2} \\
\text { sat }(\%) \dagger\end{array}$ & $A B I$ & $\begin{array}{l}P D S \\
(m m \mathrm{Hg})\end{array}$ & $\begin{array}{l}\text { Resting } \mathrm{O}_{2} \\
\text { sat (\%) }\end{array}$ & $\begin{array}{l}\mathrm{ET}(\mathrm{min}) / \mathrm{O}_{2} \\
\text { sat }(\%)\end{array}$ & $A B I$ & $\begin{array}{l}P D S \\
(m m \mathrm{Hg})\end{array}$ & & \\
\hline 1 & 69 & $100 \mathrm{~m} / 62$ & 4 & $14 / 14 / 13$ & 86 & $13 / 59$ & 2 & $20 / 18 / 18$ & $8 \times 40$ & 1.6 \\
\hline 2 & 82 & $50 \mathrm{~m} / 50$ & 4 & $20 / 11 / 13$ & 85 & No change & 4 & $17 / 13 / 16$ & $6 \times 37$ & 3.3 \\
\hline 3 & 62 & $5 / 48$ & 3 & $11 / 10 / 10$ & 83 & $7.5 / 53$ & 2 & $16 / 15 / 14$ & $6 \times 55$ & 2.9 \\
\hline 4 & 75 & $2.5 / 42$ & 3 & $20 / 5 / 11$ & 87 & $4.3 / 68$ & 2 & $33 / 21 / 26$ & $8 \times 41$ & 3.5 \\
\hline
\end{tabular}

† where exercise tolerance was very poor, metres walked was used as the exercise tolerance measure.

$\mathrm{ABI}$, ability index; ET, exercise tolerance on modified Bruce protocol or walking flat; FU, follow up; $\mathrm{O}_{2}$ sat; oxygen saturation in room air (pulse oximeter); PDS, pressure distal to stenosis; stent size $(\mathrm{mm})$, diameter $\times$ length.

haemodynamic measurements and angiography was performed in the usual way. All patients were subsequently heparinised until they were fully anticoagulated with warfarin to maintain an international normalised ratio of between 2.5 and 3.5 .

\section{Results}

The haemodynamic variables, oxygen saturation values, exercise tolerance, and follow up are given in table 2 . Four stents $(6-8 \mathrm{~mm}$ diameter, 37-55 $\mathrm{mm}$ length) were implanted. There was one procedural failure (patient 2). In this patient, stent implantation to relieve an ostial as well as multiple distal stenoses of an ascending aorta to left pulmonary artery shunt was initially satisfactory. However, during the first five to 10 minutes after implantation, the
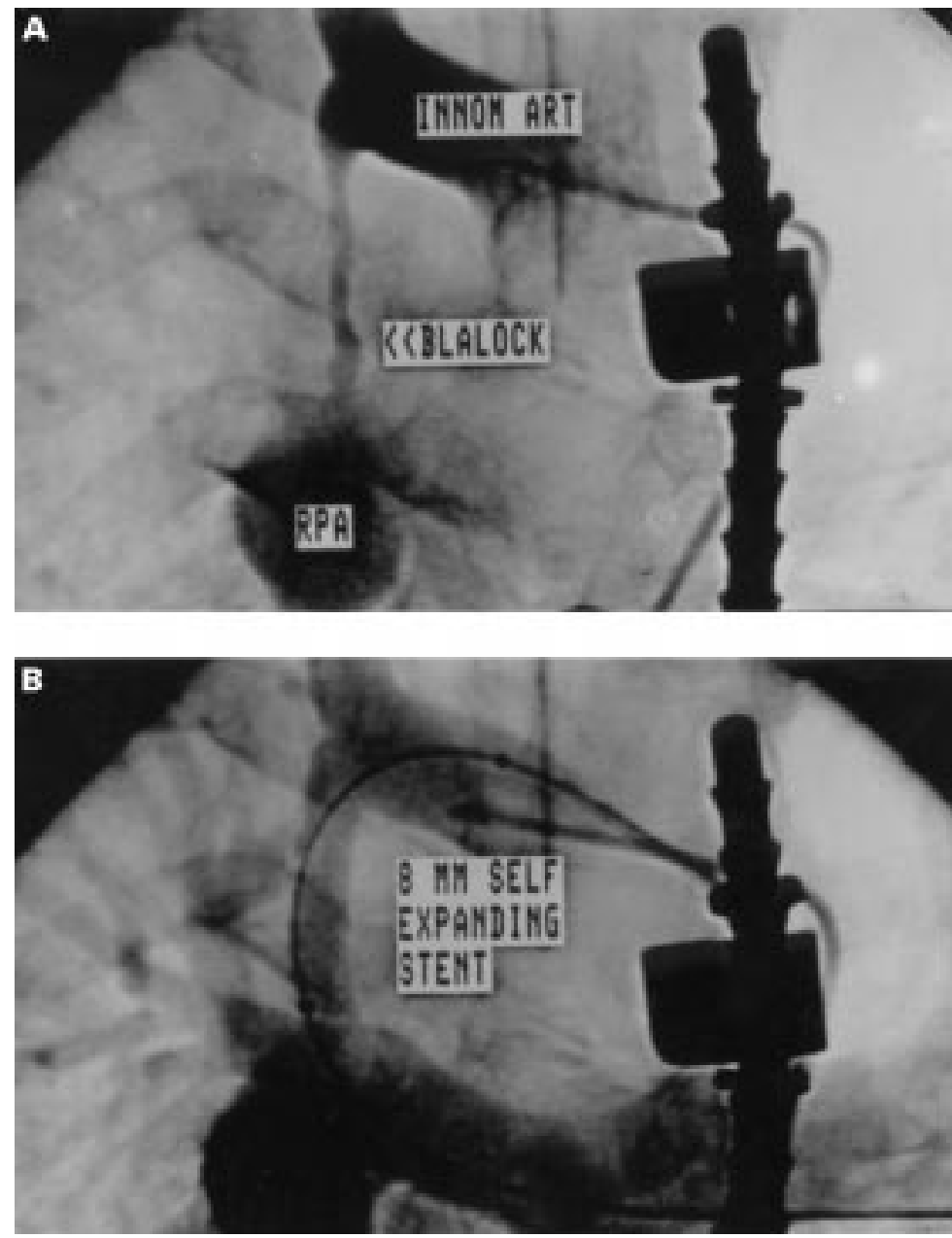

Figure 1 Angiograms done (A) before and (B) after stent insertion in patient 4. A single $(8 \mathrm{~mm} \times 4.1 \mathrm{~cm})$ self expanding stent completely relieves the multiply stenotic vessel. stent migrated distally, so that the ostial stenosis was unrelieved. Despite this there was some improvement in the distal pulmonary artery pressure, although resting oxygen saturation values were unchanged. No other procedural problems were encountered, the stenosis being completely relieved in the remainder (table 2). Pre- and postprocedural angiograms from patient 4 are shown in fig 1 .

FOLLOW UP

Follow up has extended from 1.6 to 3.5 years (table 2). Patient 2 (see above) underwent a modified Blalock-Taussig shunt distal to the migrated stent with good effect. Two patients have had a sustained, excellent symptomatic relief. One patient was non-compliant of treatment, and decreasing oxygen saturations were noted approximately six months after stent implantation. At restudy there was a 30\% reduction in stent lumen owing to thrombus. Interestingly the distal pulmonary artery pressure $(39 / 10 / 21 \mathrm{~mm} \mathrm{Hg})$ was higher than the immediate post-stent values and he has remained well palliated (resting oxygen saturation $80 \%$ at 2.9 years follow up), having reinstituted warfarin treatment.

\section{Discussion}

We describe four consecutive patients in whom stenting of a stenotic systemic arterial to pulmonary arterial shunt was attempted. There was one technical failure (case 2) in whom the stent migrated beyond the most proximal stenosis at the ostium of the shunt, at its aortic origin. In this patient the stent was placed so as to minimise the amount of stent projecting into the ascending aorta. A self expanding system was chosen because of the long segment stenosis beyond it, but with hindsight a more proximal deployment of a balloon expandable stent (into the ostial stenosis) with subsequent placement of a self expanding stent, or additional balloon expanding stents within the long segment stenosis, would have been preferable. This case illustrates one of the potential problems with self expanding stents. We presume that the rigidly stenotic nature of the ostial lesion did not allow full expansion of the stent in the desired position. Subsequently, the stent was observed to migrate from an initial ideal position to a place beyond the point of maximum stenosis. We have previously suggested that self expanding stents should ideally only be placed in lesions where complete dilatation of the stenosis is achieved with a balloon inflation pressure 
of 5 atmospheres or less. ${ }^{5}$ On this occasion, although the stenosis was balloon dilatable, the inflation pressure was not measured and this clearly was a mistake. Subsequently, however, at the patient's choice, successful surgery was performed (a modified Blalock-Taussig shunt distal to the stenosed shunt).

In the other three patients, successful deployment was associated with marked improvement in oxygen saturation and exercise tolerance. As such, the results are similar to those found in our previous study of patients with complex pulmonary atresia. ${ }^{6}$ In those patients, stenting of stenotic naturally occurring aortopulmonary collaterals also led to improvement in pulmonary blood flow and exercise tolerance. The medium term results in the current study have been excellent in the two patients who were compliant of oral anticoagulation. In the other (case 3), there was a late deterioration in oxygen saturation, coincident with failure of compliance with anticoagulation therapy. Although this patient remains well palliated, this further underscores our impression $^{6}$ that oral anticoagulation is mandatory in patients in whom a relatively small stent has been placed in areas of relatively sluggish flow and in the presence of marked erythrocytosis and cyanosis. Indeed, a similar outcome was observed in the only patient noncompliant of anticoagulation in our previous report. $^{6}$

We feel our data support the continued use of stents in this situation. While it is probable that multiple overlapping balloon expandable stents could have been used in all of these patients (in whom there were long segment and multiple stenotic lesions), self expanding stents are ideally suited for the type of stenoses often seen in these patients, with the one caveat noted in patient 2 . There remain unanswered questions, however. Even though the medium term results are promising, the long term patency of the stents, even with anticoagulation, is not yet known.

In summary, we have described the successful application of self expanding stents to the treatment of stenotic aortic to pulmonary shunts. This technique seems to have a role in the further palliation of adult patients with complex congenital heart disease.

We thank Ms Julia Peatling for her invaluable help in the preparation of this manuscript.

1 O'Laughlin MP, Perry SB, Lock-JE, et al. Use of endovascular stents in congenital heart disease. Circulation 1991;83:1923-39.

2 Mcleod KA, Blackburn ME, Gibbs JL. Stenting of stenosed aortopulmonary collaterals: a new approach to palliation in pulmonary atresia with multifocal aortopulmonary blood supply. Br Heart f 1994;71:487-9.

3 Hatai Y, Nykanen DG, Williams WG, et al. Endovascular stents in children under 1 year of age: acute impact and late results. Br Heart 7 1995; 74:689-95.

4 Zahn EM, Chang AC, Aldousany A, et al. Emergent stent placement for acute Blalock Taussig shunt obstruction after 1 stage Norwood surgery. Cathet Cardiovasc Diagn 1997;42:195.

5 Redington AN, Weil J, Somerville J. Self-expanding stents in congenital heart disease. Br Heart $\mathcal{F} 1$ 994;72;378-83.

6 Redington AN, Somerville J. Stenting of aortopulmonary collaterals in complex pulmonary atresia. Circulation 1996; 94:2479-84. 